\title{
Zur Schwefelbestimmung mittels Natriumperoxyd.
}

\author{
Von
}

Albert Neumann und Joseph Meinertz.

(Aus der chemischen Abteilung des physiologischen Instituts zu Berlin.)

(Der Redaktion zugegangen am 19. August 1904.)

In einer Abhandlung: «Über Schwefelbestimmungen in verschiedenartigen animalischen Substanzen und in Haaren von Tieren verschiedenen Alters» hat Düring ${ }^{1}$ ) die Vorzüge angegeben, welche die Substanzzerstörung mittels Soda und Natriumperoxyd vor den Methoden von Liebig und Carius hat. Er benutzt das Verfahren von Hoehnel-Glaser in der v. Asbóthschen Modifikation und erhält bei Schwefelbestimmungen in Haaren, Hufen, Fleisch sehr gut mit den älteren Methoden übereinstimmende Werte. Auch von anderen ist diese Methode mehrfach angewendet worden, so von Friedmann²) bei der Ermittelung der Konstitution des Cystins, von Sadik off ${ }^{3}$ ) bei tierischen Leimstoffen. Letzterer sieht sich bereits genötigt, die von v. Asbóth angegebenen Mengen von Soda und Peroxyd $\mathrm{zu}$ ändern, um vollständige Schmelze und Veraschung zu erzielen.

Von uns angestellte Versuche mit Hornspähnen und Casein haben nun ergeben, daß sowohl mit den ursprünglichen wie mit den von Sadikoff geänderten Mengen von Soda und Peroxyd bei Anwendung einer kleinen Spiritusflamme nur durch vielstündiges (6-8 Std.) Erhitzen eine Schmelze zu erzielen ist, während bei Zuführung größerer Hitze leicht Verpuffen oder Entzündung und somit Substanzverlust eintritt.

Will man nun das Verfahren zweckmäßig modifizieren, so hat man einerseits dafür zu sorgen, daß man selbst bei

1) Diese Zeitschrift, Bd. XXII, S. 281 (1896).

2) Beiträge zur chem. Physiol. u. Pathol., Bd. 3, S. 1 (1902).

$\left.{ }^{3}\right)$ Diese Zeitschrift, Bd. XXXIX, S: 396 (1903). 
mäßiger Flamme leicht eine Schmelze erzielt, und andererseits die Mengen und den Zusatz des Peroxyds so zu regulieren, daß Verpuffen und Entzündung des Veraschungsgemischs ausgeschlossen ist. Das letztere erreicht man leicht, wenn man das Peroxyd nicht mit einem Male, sondern portionsweise (etwa 3 Portionen) zusetzt; für den ersteren Punkt dagegen war die Tatsache maßgebend, daß Kaliumnatriumkarbonat viel schneller schmilzt als Soda. Wie bekannt, verwendet man deshalb auch bei der Aufschließung von Silicaten u.s.w. diese Kalinatronverbindung viel zweckmäßiger als einfaches Natriumkarbonat. Es scheint überhaupt, daß die Anwesenheit von Kali neben Natron bei solchen Schmelzen und Veraschungen einen wesentlichen Vorteil bietet. Unsere Versuche haben nun ergeben, daß man, wenn man das Verfahren nach den obigen Gesichtspunkten modifiziert, auch bei sehr schwer verbrennlichen Substanzen wie Casein und Hornspähnen verhältnismäßig schnell zum Ziele kommt und sehr gute Resultate erhält.

Sowohl bei der Veraschung mit Peroxyd nach Asbóth wie bei der Liebigschen Substanzzerstörung durch Ätzalkali und Salpeter ist die Benutzung einer Spiritusflamme vorgeschrieben. Durch unsere Versuche konnte dagegen festgestellt werden, daß durch die Anwendung eines Bunsenschen Gasbrenners keinerlei Fehler entstehen. Bei der von uns vorgeschlagenen Modifikation ist sogar eine Gasflamme erforderlich, weil am Ende der Veraschung eine solche Temperatur nötig ist, wie sie eine Spiritusflamme nicht erzeugt.

Ausfïhrung der Bestimmung: $1 \mathrm{~g}$ Substanz (z. B. Casein) wird mit $5 \mathrm{~g}$ Kaliumnatriumkarbonat und $2^{1 / 2} \mathrm{~g}$ Natriumperoxyd in einen Nickeltiegel von etwa $100 \mathrm{ccm}$ Inhalt innig vermengt und über einer kleinen Gasflamme ungefähr 1 Stunde lang erhitzt, bis die Mischung völlig zusammengesintert ist. Nach kurzer Abkühlung (etwa 5 Minuten) werden wieder 21/2 g Peroxyd zugesetzt; dann wird mit kleiner Flamme noch einmal etwa 1 Stunde erwärmt, und zwar bis die Hauptmenge sich verflüssigt hat. Hierauf entfernt man den Gasbrenner, gibt noch $2 \mathrm{~g}$ Peroxyd hinein und glüht ca. 1/4 Stunde, indem man die Flamme allmählich bis zur vollen Stärke vergrößert. Alsdann ist völlige 
Verflüssigung eingetreten. - Der Tiegel bleibt dauernd bedeckt. Man kann die Schmelze während der ganzen Veraschung sich selbst überlassen; Verpuffen und Entzündung der Substanz sind sicher $\mathrm{zu}$ vermeiden, wenn man nur darauf achtet, daß man die Gasflamme bis zur letzten Viertelstunde, besonders aber am Anfang, nicht zu groß macht.

Die erkaltete Schmelze hat infolge geringer Mengen beigemengten Nickeloxyds eine grünlich-graue Farbe. Sie wird im Tiegel mit Wasser übergossen und bedeckt (wegen der Gasentwickelung) mit kleiner Flamme bis zur Lösung erhitzt. Die Flüssigkeit wird in ein Becherglas übergespült, mit bromhaltiger Salzsäure vorsichtig (Bedecken mit einem Uhrglas) sauer gemacht und nun auf dem Wasserbade einige Zeit erhitzt, wobei eine klare, grünliche Lösung entsteht. Da bei diesem Verfahren die Substanz vollständig verbrennt, so bleibt keine Kohle zurück, Filtrieren ist daher unnötig. Sodann wird in der üblichen Weise heiß mit Chlorbaryum gefällt, filtriert und das Baryumsulfat nach dem Glühen gewogen.

Bei leicht verbrennlichen Substanzen muß man besonders zu Anfang weniger Peroxyd (etwa $1 \mathrm{~g}$ statt $2^{1 / 2} \mathrm{~g}$ ) nehmen.

Resultate: Zur Prüfung der Methode wurden folgende Bestimmungen ausgeführt:

a) Zunächst wurde eine Mischung von $1 \mathrm{~g}$ reinem Traubenzucker und $10 \mathrm{ccm}$ einer Schwefelnatriumlösung, deren Schwefelgehalt $0,1302 \mathrm{BaSO}_{4}$ entsprach, nach dem vorbeschriebenen Verfahren behandelt; die hierbei gefundene Schwefelmenge entsprach 0,1307 $\mathrm{BaSO}_{4}$, war also mit der ohne Veraschung ermittelten übereinstimmend.

Dieses Resultat hat aber insofern noch ein Interesse, weil es unter Benutzung einer Gasflamme erhalten worden ist und zeigt, daß man unter diesen Umständen keine höheren Werte bekommt. Die Hitze einer Spiritusflamme dagegen genügte bei der angegebenen Modifikation nicht. Der gleiche Ansatz der oben beschriebenen Mischung von Traubenzucker und Schwefelnatrium zeigte sich unter Benutzung einer Spiritusflamme nicht vollständig verascht, da er beim Auflösen Kohle hinterließ und einen Schwefelgehalt ergab, welcher nur $0,1262 \mathrm{BaSO}_{4}$ entsprach. 
40 Alb. Neumann u. Jos. Meinertz, Zur Schwefelbestimmung.

b) Die folgenden Resultate wurden mit käuflichem Casein und fein zerriebenen Hornspähnen erhalten.

Casein.

1,2135 g Substanz gaben $0,0653 \mathrm{~g} \mathrm{BaSO}_{4}=0,7389 \% \mathrm{~S}$

1,0195 > > $>0,0550$ > > $=0,7411 \%$,

1,0410 > > 0,0559 > > $=0,7374 \%$ ।

Hornspähne.

$0,4928 \mathrm{~g}$ Substanz gaben $0,1055 \mathrm{~g} \mathrm{BaSO}_{4}=2,940 \% \mathrm{~S}$

0,5103 > > 0,1092 > > $=2,938 \%$, 